\title{
Theory of grating-coupled excitation of Dyakonov surface waves (Erratum)
}

\section{Kiran Mujeeb, ${ }^{a}$ Muhammad Faryad, ${ }^{b}$ Akhlesh Lakhtakia, ${ }^{c}$ and Julio V. Urbina ${ }^{\mathrm{d}, *}$ \\ ${ }^{a}$ Quaid-i-Azam University, Department of Electronics, Islamabad, Pakistan}

${ }^{\mathrm{b}}$ Lahore University of Management Sciences, Department of Physics, Lahore, Pakistan

${ }^{\mathrm{c}}$ The Pennsylvania State University, Department of Engineering Science and Mechanics, University Park, Pennsylvania, United States

${ }^{\mathrm{d}}$ The Pennsylvania State University, Department of Electrical Engineering, University Park, Pennsylvania, United States

[DOI: 10.1117/1.OE.60.6.069801]

This letter [Optical Engineering, 59(7), 070503 (2020) DOI: 10.1117/1.OE.59.7.070503] was originally published on 24 July 2020 with an error in Eq. (4).

The incorrect equation appeared as:

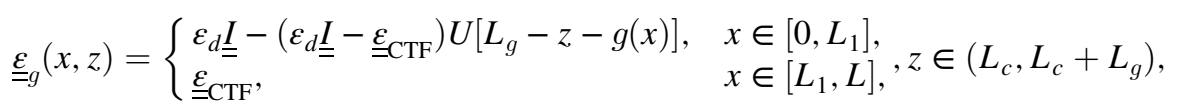

The corrected equation appears as:

$$
\underline{\underline{\varepsilon}}_{g}(x, z)= \begin{cases}\varepsilon_{d} \underline{\underline{I}}-\left(\varepsilon_{d} \underline{\underline{I}}-\underline{\underline{\varepsilon}}_{\mathrm{CTF}}\right) U\left[L_{g}+L_{c}-z-g(x)\right], & x \in\left[0, L_{1}\right], \\ \underline{\underline{\varepsilon}}_{\mathrm{CTF}}, & x \in\left[L_{1}, L\right], \quad z \in\left(L_{c}, L_{c}+L_{g}\right) .\end{cases}
$$

Also, in the first paragraph in Sec. 3.1, the conditions on imaginary part of $\alpha$ were incorrectly stated. The correct conditions are $\operatorname{Im}(\alpha)<0$ in the $\mathrm{CTF}$ and $\operatorname{Im}(\alpha)>0$ in the isotropic dielectric material.

These errors were not present in the computations and all results in the letter remain correct.

The paper was corrected on 24 June 2021.

*Address all correspondence to Julio V. Urbina, E-mail: jvu1@psu.edu 